\title{
The effect of neoadjuvant short-course radiotherapy and delayed surgery versus chemoradiation on postoperative outcomes in locally advanced rectal cancer patients - A propensity score matched nationwide audit-based study
}

\author{
S. Hoendervangers a, b, *, C.L. Sparreboom ${ }^{c}$, M.P.W. Intven ${ }^{\text {a }}$, J.F. Lange ${ }^{c, d}$, \\ H.M. Verkooijen ${ }^{\mathrm{a}}$, P.G. Doornebosch ${ }^{\mathrm{d}}$, \\ W.M.U. van Grevenstein ${ }^{\mathrm{b}}$, on behalf of the Dutch ColoRectal Audit \\ a Department of Radiation Oncology, University Medical Center Utrecht, Utrecht, the Netherlands \\ b Department of Surgery, University Medical Center Utrecht, Utrecht, the Netherlands \\ ${ }^{\mathrm{c}}$ Department of Surgery, Erasmus MC, Rotterdam, the Netherlands \\ ${ }^{\mathrm{d}}$ Department of Surgery, IJsselland Ziekenhuis, Capelle a/d Ijssel, the Netherlands
}

\section{A R T I C L E I N F O}

\section{Article history:}

Accepted 3 March 2020

Available online $\mathrm{xxx}$

\section{Keywords:}

Short-course radiotherapy

Delayed surgery

Chemoradiation

Postoperative outcomes

Locally advanced rectal cancer

\begin{abstract}
A B S T R A C T
Objective: To investigate differences in postoperative outcomes between short-course radiotherapy and delayed surgery (SCRT-delay) and chemoradiation (CRT) in patients with locally advanced rectal cancer (LARC).

Background: Previous trials suggest that SCRT-delay could serve as an adequate neoadjuvant treatment for LARC. Therefore, in frail LARC patients SCRT-delay is recommended as an alternative to CRT. However, data on postoperative outcomes after SCRT-delay in comparison to CRT is scarce.

Methods: This was an observational study with data from the Dutch ColoRectal Audit (DCRA). LARC patients who underwent surgery (2014-2017) after an interval of $\geq 6$ weeks were included. Missing values were replaced by multiple imputation. Propensity score matching (PSM), using age, Charlson Comorbidity Index, cT-stage and surgical procedure, was applied to create comparable groups. Differences in postoperative outcomes were analyzed using Chi-square test for categorical variables, independent sample $t$-test for continuous variables and Mann-Whitney $U$ test for non-parametric data. Results: 2926 patients were included. In total, 288 patients received SCRT-delay and 2638 patients underwent CRT. Patients in the SCRT-delay group were older and had more comorbidities. Also, ICUadmissions and permanent colostomies were more common, as well as pulmonic, cardiologic, infectious and neurologic complications. After PSM, both groups comprised 246 patients with equivalent age, comorbidities and tumor stage. There were no differences in postoperative complications.

Conclusion: Postoperative complications were not increased in LARC patients undergoing SCRT-delay as neoadjuvant treatment. Regarding treatment-related complications, SCRT-delay is a safe alternative neoadjuvant treatment option for frail LARC patients.
\end{abstract}

๑ 2020 Elsevier Ltd, BASO The Association for Cancer Surgery, and the European Society of Surgical Oncology. All rights reserved.

\section{Introduction}

In compliance with European guidelines, neoadjuvant treatment for locally advanced rectal cancer (LARC) in the Netherlands

\footnotetext{
* Corresponding author. Q00.311, P.O. Box 855003508 GA Utrecht, Netherlands

E-mail address: s.hoendervangers@umcutrecht.nl (S. Hoendervangers).
}

comprises neoadjuvant chemoradiation (CRT), followed by surgery according to total mesorectal excision (TME) principles. Shortcourse radiotherapy followed by surgery after a prolonged interval (SCRT-delay) is recommended as an alternative to chemoradiation in older patients with comorbidities or frail patients with a poor performance status, because of their higher risk of treatment related complications [1].

Postoperative morbidity and mortality are often increased in

https://doi.org/10.1016/j.ejso.2020.03.002

0748-7983/@ 2020 Elsevier Ltd, BASO The Association for Cancer Surgery, and the European Society of Surgical Oncology. All rights reserved. 
frail or elderly patients as a result of concomitant comorbidities [2]. Data on surgical management of rectal cancer in these patients is scarce and reported postoperative morbidity and mortality vary widely in the population $>65$ years old [2-4]. This patient group might benefit from altered treatment, especially when they are more susceptible to treatment-related complications [5]. Moreover, inadequate treatment is associated with poor survival [2]. Unfortunately, the heterogeneity of this group and the lack of data impede an evidence-based choice of neoadjuvant treatment in this patient group [6]. With the aging population, there is need for evidence to justify the choice of the most optimal neoadjuvant treatment in frail patients with LARC.

Previous trials suggest that SCRT-delay could serve as an adequate neoadjuvant treatment for intermediate to high risk rectal cancer [7-9]. Although previous trials showed that an interval $<10$ days between SCRT and surgery is associated with anastomotic leakage and postoperative mortality [10,11], the rate of postoperative complications in the Stockholm III trial was lower when surgery was delayed for 4-12 weeks after SCRT [12], suggesting that it is better to prolong the interval between SCRT and surgery. Before adding this regimen to current guidelines, more data is needed on postoperative outcomes of SCRT-delay in comparison to CRT. The aim of this study was to investigate the effect of SCRT-delay on postoperative outcomes in comparison with CRT, in both the general and the frail population.

\section{Methods}

\section{Study design}

This was an observational study with data from the Dutch ColoRectal Audit (DCRA), a nationwide audit which registers clinical outcomes of all patients undergoing primary colorectal surgery in the Netherlands. The DCRA is based on evidence-based guidelines and is validated on a yearly basis with data from the Netherlands Cancer Registry (NCR) [13]. Because data could not be traced back to individual patients, neither informed consent nor ethical approval was required for this study.

\section{Patient selection}

All patients with $\geq \mathrm{cT} 2$ rectal cancer who underwent surgery between May 2014 (after implementation of a new Dutch colorectal cancer guideline) and December 2017, were selected from the DCRA database. Based on Dutch guidelines, LARC was defined as cT4, $\mathrm{CT}_{\text {any }}$ with mesorectal fascia (MRF) involvement, or $\mathrm{CT}_{\text {any }} \mathrm{N} 2$. All patients with LARC were included in the study. Clinical tumor stage was based on imaging. Patients were excluded in case of metastatic disease, tumors located outside the rectum, emergency or urgent surgery. Also, patients who did not receive neoadjuvant treatment, with a missing start date of neoadjuvant therapy, or patients who underwent surgery after an interval of less than 6 weeks after the end of radiotherapy were excluded. Furthermore, patients who underwent surgery after an initial watch and wait strategy were excluded from the dataset, since the prolonged interval in this group could be associated with higher morbidity and a more difficult surgical resection [14]. Finally, patients who received intraoperative Hyperthermic Intraperitoneal Chemotherapy (HIPEC) or Intraoperative Radiation Therapy (IORT) were excluded.

\section{Neoadjuvant therapy}

Neoadjuvant treatment entailed either SCRT (25 Gy in fractions of $5 \mathrm{~Gy}$ in 5 days) or CRT (45-50 Gy in fractions of $1.8-2 \mathrm{~Gy}$ in 5 weeks and concurrent oral Capecetabine $825-1000 \mathrm{mg} / \mathrm{m} 2$ twice daily 5-7 days a week). Interval to surgery was calculated from the end of neoadjuvant treatment. The interval between the end of SCRT and surgery was calculated by subtracting 4 days from the interval when treatment started on Monday or by subtracting 6 days from the interval when treatment started on another day, accounting for discontinuation of therapy in the weekend. The same syntax was used for CRT patients, subtracting 32 or 34 days, respectively.

\section{Data collection}

Patient characteristics included gender, age at surgery, BMI (kg/ $\mathrm{m}^{2}$ ), number and type of comorbidities, and ASA score. Charlson Comorbidity Index was calculated according to the weighted index of comorbidity [15]. Tumor characteristics included clinical TNMstage, MRF involvement and tumor distance from the anus (measured at colonoscopy). Treatment characteristics included type of neoadjuvant treatment (SCRT or CRT), date of surgery, surgical procedure and approach, intraoperative complications, conversion, and ostomy creation. The subgroup 'Minimally invasive approaches' included transanal endoscopic microsurgery (TEM), local excision and transanal minimally invasive local excision (TAMIS). Hartmann procedure was incorporated in the subgroup '(Low) Anterior Resection'. Subtotal colectomy, proctocolectomy and sigmoid resection were combined in the subgroup 'other surgical procedures' because of low prevalence. Intraoperative complications comprised injury of intra-abdominal structures, complications requiring blood transfusion or other non-specified complications.

\section{Outcome measures}

Follow-up time was 30 days after surgery. The primary outcome measure was the occurrence of postoperative complications. Complications were defined according to standards of the DCRA [13]. Postoperative complications comprised both surgical and nonsurgical complications and were defined as hospital stay of $\geq 14$ days and/or a complication, re-intervention due to a complication, and/or death during hospital stay or within 30 days after surgery. Postoperative surgical complications included anastomotic failure, abscess, bleeding, ileus, dehiscent fascia, iatrogenic bowel injury, ureter/urethra injury, or other non-specified complications. Postoperative non-surgical complications included pulmonary, cardiac, thrombotic, infectious, neurologic or other non-specified complications.

Postoperative outcome measures included re-intervention, prolonged hospital stay, intensive care unit (ICU) stay and readmission. Re-intervention involved any laparotomy-, laparoscopic- or radiology-assisted treatment for a complication. Admission to the ICU and length of hospital stay were dichotomized based on the median length of admission. ICU stay was defined as admission to the ICU for at least 1 day. Prolonged hospital stay was defined as admission to the surgical ward for more than 7 days. Pathological outcomes included pathological tumor and nodal stage, pathological complete response rate (ypTONO) and resection margin.

\section{Missing data}

On average, there was $2.3 \%$ missing data. Missing values were classified as random and replaced by multiple imputation. All observed data, including the outcome, that were applied to the dataset after imputation were used as predictors [16,17]. The number of imputations depended on the average percentage rate of missingness [18,19]. The imputed data were checked with 
convergence plots. Imputation was successful if the streams intermingled and were free of any trend [17]. Finally, 5 imputed datasets were produced with 5 iterations.

\section{Propensity score matching}

Since frail patients are more likely to receive SCRT-delay or to experience postoperative complications, and the type of surgery is a determinant of postoperative complications, the likelihood of confounding by indication needed to be accounted for. To enable a comparison in equivalent groups, propensity score matching was performed [20]. The propensity score was calculated for each patient using logistic regression with the variables age, Charlson Comorbidity Index, cT-stage, and surgical procedure. These covariates were chosen based on their clinical relevance. Propensity score matching was performed using 'nearest-neighbor matching' without replacement and a 1:1 ratio. The average within-pair difference in propensity scores was minimized by setting a caliper of 0.25 multiplied by the standard deviation of the logit of the propensity score [21]. The balance in the matched dataset was expressed in 'standardized mean difference' (SMD), with an SMD $<0.10$ indicating a well-balanced set [22-24].

\section{Statistical analyses}

Differences in baseline characteristics and treatment outcomes were analyzed using Chi-square test for categorical variables and independent sample $t$-test for continuous variables. MannWhitney $U$ test was used for non-parametric data. The Bonferroni correction was applied to account for multiple testing. The level of significance was set at $\mathrm{p}<0.05$.

All analyses were performed in IBM SPSS Statistics (version 23 - (C) 2015 IBM Corporation) and RStudio (Version 1.0.143 - (c) 2009-2016 RStudio, Inc.,'mice', 'tableone', 'MatchIt' and 'optmatch' packages).

\section{Results}

\section{Patient selection}

Between May 2014 and December 2017, 8318 patients with $\geq$ cT2 rectal cancer were registered in the DCRA database. Patients without locally advanced tumors $(\mathrm{n}=3541)$, with metastatic disease $(n=986)$, with tumors located outside the rectum $(n=257)$, who underwent urgent or emergency surgery $(n=15)$, who did not receive neoadjuvant treatment or if the start date of neoadjuvant therapy was missing ( $n=31$ ), who received chemotherapy only or chemotherapy combined with short-course radiotherapy $(n=102)$, who underwent surgery after an interval of less than 6 weeks after the end of radiotherapy $(n=298)$ or after an initial watch and wait strategy $(\mathrm{n}=34)$ and who underwent HIPEC or IORT $(\mathrm{n}=128)$ (Supplementary Fig. 1) were excluded. Finally, 2926 patients were included in the analysis. Non-imputed data are provided in Supplementary Tables 1 and 2

\section{Pre-matching results}

Patients in the SCRT-delay group had a higher mean age, had more comorbidities and a higher ASA score (Table 1). Mean BMI was higher in the CRT group. The MRF was less often involved in the SCRT-delay group and clinical N-stage was higher. There was no difference in the distance from the anus at colonoscopy. With an equal interval between neoadjuvant treatment and surgery, patients in the SCRT-delay group more often underwent abdominoperineal resection (APR) and more often received a permanent colostomy. In the SCRT-delay group, 63 patients (21.9\%) received a primary anastomosis, compared to 1253 patients (47.5\%) in the CRT group. Pulmonic, cardiologic, infectious and neurologic complications were significantly more common in the SCRT-delay group (Table 2). When stratified for procedure, there were significant more complications after APR in the SCRT-delay group; however, pulmonic, cardiologic, infectious and neurologic complications in the SCRT-delay group occurred independent of type of surgical procedure (Supplementary Table 3 ). There was no difference in the number of re-interventions. In patients that received a primary anastomosis, the frequency of re-interventions for anastomotic leakage was not different after SCRT-delay or CRT (6.3\% vs $7.6 \%$, respectively). Patients in the SCRT-delay group were more often admitted to the ICU and hospital stay was more often prolonged. Furthermore, SCRT-delay less often resulted in a pathological complete response compared to CRT ( $8.0 \%$ vs. $16.1 \%$, Table 1$)$. There were no differences in surgical radicality after SCRT-delay or CRT (92.7\% vs. 95.2\% R0 resections, respectively).

\section{Post-matching results}

Baseline characteristics that entered the propensity score model are presented in Supplementary Table 4. After matching, both groups comprised 246 patients and characteristics were wellbalanced. Differences in patient, treatment and pathological characteristics between SCRT-delay and CRT in the post-matching cohort are presented in Table 3. After matching, BMI was higher in the CRT group. 57 patients in the SCRT-delay group (23.2\%) received a primary anastomosis, compared to 73 patients $(29.7 \%)$ in the CRT group. Permanent colostomies were more frequent in the SCRT-delay group, but this difference was not significant in the post-matching cohort. There were no differences in pathological outcomes. Overall, there were no differences in postoperative (surgical) complications (Table 4). The number of re-interventions for anastomotic leakage in patients that received a primary anastomosis was not significantly different between groups (5.3\% vs. $2.7 \%$ after SCRT-delay vs. CRT, respectively). There were no differences in ICU admission and hospital stay.

\section{Discussion}

In this nationwide, propensity score matched study we found no difference in the occurrence of surgical complications between patients who underwent SCRT-delay or CRT as neoadjuvant therapy for LARC. However, more pulmonic, cardiologic, infectious and neurologic complications in the pre-matching cohort in the SCRTdelay group. These differences diminished when patients were matched on age, gender, comorbidities, tumor characteristics and distance from the anus.

The pre-matching cohort represents daily clinical practice in the Netherlands. With the addition of SCRT-delay as regimen for LARC to the Dutch guidelines in 2014, more elderly patients are offered neoadjuvant treatment [25]. In our dataset, 642 of 2926 (21.9\%) patients who underwent surgery were aged $\geq 75$ years. The percentage of old and frail patients was higher in the SCRT-delay group, but these patients were also represented in the CRT group, which underlines the heterogeneity of the elderly population and the differences in treatment choice due to lack of evidence-based data. Previous studies showed various results in incidence of postoperative morbidity and mortality in the elderly [2-5,26-28]. Taking into account that mortality increases when postoperative complications occur [5,27] and anastomotic leakage results in significantly more anorectal and urinary symptoms and higher Low Anterior Resection Syndrome (LARS) scores [29,30], the lower prevalence of primary anastomosis in the SCRT-delay group in the unmatched cohort might be a result of a defensive attitude towards 
Table 1

Patient, tumor and pathological characteristics in the pre-matching cohort. Data are presented as number (percentage) unless stated otherwise.

\begin{tabular}{|c|c|c|c|}
\hline & SCRT-delay $\mathrm{n}=288$ & CRT $\mathrm{n}=2638$ & p-value \\
\hline \multicolumn{4}{|l|}{ Patient characteristics } \\
\hline Gender & & & $0.021 *$ \\
\hline Male & $161(55.9)$ & $1663(63.0)$ & \\
\hline Female & $127(44.1)$ & $975(37.0)$ & \\
\hline Age (mean years $(\mathrm{sd})$ ) & $77.89(8.76)$ & $64.59(10.25)$ & $<0.001 *$ \\
\hline BMI $\left(\right.$ mean $\left.\mathrm{kg} / \mathrm{m}^{2}(\mathrm{sd})\right)$ & $25.54(3.94)$ & $26.27(4.35)$ & $0.006 *$ \\
\hline Comorbidities & $249(86.5)$ & $1765(66.9)$ & $<0.001 *$ \\
\hline Charlson Comorbidity Index & & & $<0.001 *$ \\
\hline 0 & $103(35.8)$ & $1645(62.4)$ & \\
\hline 1 & $85(29.5)$ & $554(21.0)$ & \\
\hline 2 & $48(16.7)$ & $277(10.5)$ & \\
\hline 3 & $31(10.8)$ & $111(4.2)$ & \\
\hline$\geq 4$ & $21(7.3)$ & $51(1.9)$ & \\
\hline ASA score & & & $<0.001 *$ \\
\hline 1 & 20 (6.9) & $575(21.8)$ & \\
\hline 2 & $157(54.5)$ & $1686(63.9)$ & \\
\hline 3 & $103(35.8)$ & $363(13.8)$ & \\
\hline 4 & $8(2.8)$ & $14(0.5)$ & \\
\hline \multicolumn{4}{|l|}{ Tumor characteristics } \\
\hline Distance from anus at colonoscopy (mean $\mathrm{cm}(\mathrm{sd})$ ) & $6.69(4.69)$ & $6.36(4.42)$ & 0.224 \\
\hline cT & & & 0.425 \\
\hline 2 & $8(2.8)$ & $95(3.6)$ & \\
\hline 3 & $225(78.1)$ & $1971(74.7)$ & \\
\hline 4 & $55(19.1)$ & $572(21.7)$ & \\
\hline Distance to MRF $>1 \mathrm{~mm}$ & $58(20.1)$ & $721(27.3)$ & $0.003 *$ \\
\hline cN & & & $<0.001 *$ \\
\hline 0 & $77(26.7)$ & $346(13.1)$ & \\
\hline 1 & $80(27.8)$ & $648(24.6)$ & \\
\hline 2 & $129(44.8)$ & $1637(62.1)$ & \\
\hline$x$ & $2(0.7)$ & $7(0.3)$ & \\
\hline \multicolumn{4}{|l|}{ Treatment characteristics } \\
\hline Interval between end of neoadjuvant treatment and surgery (median weeks [IQR]) & $11.00[9.00,15.00]$ & $11.00[10.00,13.00]$ & 0.472 \\
\hline Surgical approach & & & $<0.001 *$ \\
\hline Transabdominal open & $58(20.1)$ & $561(21.3)$ & \\
\hline Transabdominal scopic & $204(70.8)$ & $1933(73.3)$ & \\
\hline TaTME or TAMIS TME & $18(6.2)$ & $134(5.1)$ & \\
\hline Minimally invasive & $8(2.8)$ & $10(0.4)$ & \\
\hline Surgical procedure & & & $<0.001 *$ \\
\hline Local excision & $8(2.8)$ & $6(0.2)$ & \\
\hline (Low) Anterior Resection & $155(53.8)$ & $1607(60.9)$ & \\
\hline Abdominoperineal Resection & $124(43.1)$ & $996(37.8)$ & \\
\hline Other & $1(0.3)$ & $29(1.1)$ & \\
\hline Conversion & $39(13.5)$ & $341(12.9)$ & 0.839 \\
\hline Reason conversion & & & 0.658 \\
\hline Extensive tumor growth & $8(2.8)$ & $6(0.2)$ & \\
\hline Accessibility & $28(9.7)$ & $216(8.2)$ & \\
\hline Intraoperative complication & $3(1.0)$ & $47(1.8)$ & \\
\hline Intraoperative complication & & & 0.954 \\
\hline No & $275(95.5)$ & $2506(95.0)$ & \\
\hline Bleeding & $1(0.3)$ & $17(0.6)$ & \\
\hline Spleen injury & $0(0.0)$ & $1(0.0)$ & \\
\hline Bowel injury & $3(1.0)$ & $19(0.7)$ & \\
\hline Ureter/urethra injury & $4(1.4)$ & $26(1.0)$ & \\
\hline Bladder injury & $1(0.3)$ & $17(0.6)$ & \\
\hline Vagina injury & $1(0.3)$ & $12(0.5)$ & \\
\hline Other & $3(1.0)$ & $40(1.5)$ & \\
\hline Primary anastomosis & $64(22.2)$ & $1257(47.6)$ & $<0.001 *$ \\
\hline Ostomy & & & $<0.001 *$ \\
\hline No & $57(19.8)$ & $521(19.7)$ & \\
\hline Diverting ileostomy & $27(9.4)$ & $773(29.3)$ & \\
\hline Permanent ileostomy & $1(0.3)$ & $29(1.1)$ & \\
\hline Diverting colostomy & $7(2.4)$ & $125(4.7)$ & \\
\hline Permanent colostomy & $195(67.7)$ & $1186(45.0)$ & \\
\hline Stoma, unknown type & $1(0.3)$ & $4(0.2)$ & \\
\hline \multicolumn{4}{|l|}{ Pathological characteristics } \\
\hline pT & & & $0.001 *$ \\
\hline 0 & $27(9.4)$ & $512(19.4)$ & \\
\hline 1 & $15(5.2)$ & $157(6.0)$ & \\
\hline 2 & $75(26.0)$ & $623(23.6)$ & \\
\hline 3 & $152(52.8)$ & $1202(45.6)$ & \\
\hline 4 & $19(6.6)$ & $125(4.7)$ & \\
\hline
\end{tabular}

Please cite this article as: Hoendervangers $S$ et al., The effect of neoadjuvant short-course radiotherapy and delayed surgery versus chemoradiation on postoperative outcomes in locally advanced rectal cancer patients - A propensity score matched nationwide audit-based study, European Journal of Surgical Oncology, https://doi.org/10.1016/j.ejso.2020.03.002 
Table 1 (continued)

\begin{tabular}{|c|c|c|c|}
\hline & SCRT-delay $\mathrm{n}=288$ & CRT $n=2638$ & p-value \\
\hline$x$ & $0(0.0)$ & $13(0.5)$ & \\
\hline Unknown & $0(0.0)$ & $6(0.2)$ & \\
\hline pN & & & 0.831 \\
\hline 0 & $185(64.2)$ & $1776(67.3)$ & \\
\hline 1 & $67(23.3)$ & $571(21.6)$ & \\
\hline 2 & $35(12.2)$ & $282(10.7)$ & \\
\hline$x$ & $1(0.3)$ & $7(0.3)$ & \\
\hline Unknown & $0(0.0)$ & $2(0.1)$ & \\
\hline Pathological complete response, ypTONO & $23(8.0)$ & $425(16.1)$ & $<0.001 *$ \\
\hline Radicality ( $\mathrm{RO}$ resection) & $267(92.7)$ & $2512(95.2)$ & 0.087 \\
\hline
\end{tabular}

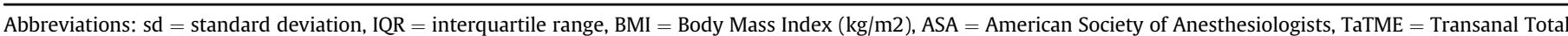
Mesorectal Excision, TAMIS TME = TransAnal Minimal Invasive Surgery - Total Mesorectal Excision, $\mathrm{pT}=$ pathological tumor stage, $\mathrm{pN}=$ pathological nodal stage.

Table 2

Postoperative outcomes in the pre-matching cohort. Data are presented as number percentage) unless stated otherwise.

\begin{tabular}{|c|c|c|c|}
\hline & SCRT-delay $\mathrm{n}=288$ & CRT $n=2638$ & p-value \\
\hline Any postoperative complication $<\mathbf{3 0}$ days after surgery & $124(43.1)$ & $987(37.4)$ & 0.070 \\
\hline \multicolumn{4}{|l|}{ Non-surgical complications $<\mathbf{3 0}$ days after surgery } \\
\hline Pulmonic & $25(8.7)$ & $102(3.9)$ & $<0.001 *$ \\
\hline Cardiologic & $18(6.2)$ & $71(2.7)$ & $0.002 *$ \\
\hline Infectious & $32(11.1)$ & $136(5.2)$ & $<0.001 *$ \\
\hline Thrombotic & $1(0.3)$ & $14(0.5)$ & 1.000 \\
\hline Neurological & $12(4.2)$ & $38(1.4)$ & $0.002 *$ \\
\hline Other & $48(16.7)$ & $360(13.6)$ & 0.188 \\
\hline Surgical complications $<\mathbf{3 0}$ days after surgery & $66(22.9)$ & $653(24.8)$ & 0.538 \\
\hline Re-intervention & $31(10.8)$ & $317(12.0)$ & 0.598 \\
\hline Type of re-intervention & & & 0.334 \\
\hline Radiologic & $2(0.7)$ & $35(1.3)$ & \\
\hline Surgery, laparoscopic & $3(1.0)$ & $69(2.6)$ & \\
\hline Surgery, open & $19(6.6)$ & $138(5.2)$ & \\
\hline Other & $7(2.4)$ & $76(2.9)$ & \\
\hline Reason re-intervention & & & 0.572 \\
\hline Anastomotic failure ${ }^{\mathrm{a}}$ & 4 of $64(6.3)$ & 96 of $1257(7.6)$ & \\
\hline Abscess & $9(3.1)$ & $74(2.8)$ & \\
\hline Bleeding & $1(0.3)$ & $11(0.4)$ & \\
\hline Ileus & $2(0.7)$ & $40(1.5)$ & \\
\hline Fascial dehiscence & $2(0.7)$ & $17(0.6)$ & \\
\hline Iatrogenic bowel injury & $0(0.0)$ & $5(0.2)$ & \\
\hline Ureter/urethra injury & $2(0.7)$ & $5(0.2)$ & \\
\hline Other & $9(3.1)$ & $62(2.4)$ & \\
\hline$\geq 1$ day on $\mathbf{I C U}$ & $98(34.0)$ & $712(27.0)$ & $0.014 *$ \\
\hline Hospital stay $\geq 7$ days & $145(50.3)$ & $1076(40.8)$ & $0.002 *$ \\
\hline Readmission $<30$ days after surgery & $36(12.5)$ & $384(14.6)$ & 0.539 \\
\hline Death during or $\leq \mathbf{3 0}$ days after surgery & $4(1.4)$ & $23(0.9)$ & 0.268 \\
\hline
\end{tabular}

Abbreviations: ICU = intensive care unit.

a Data shown for patients that received a primary anastomosis.

primary anastomoses in frail and older patients. More postoperative complications were seen in this cohort after APR in the SCRT-delay group. However, the low prevalence indicates that conclusions should be drawn cautiously. Also, the rate of pulmonic, cardiologic, infectious and neurologic complications was higher in this group. However, this was not related to surgical procedure and can therefore most likely be explained by the frailty of the SCRTdelay population. The lack of differences in surgical complications can partly be explained by improved quality of care and better selection of patients $[6,31]$. Moreover, frail patients that did not undergo surgical resection were not included in this study.

In the pre-matching cohort we found a significant lower $\mathrm{pCR}$ rate in the SCRT-delay group ( $8.0 \%$ vs. $16.1 \%$ ) after a median interval to surgery of 11 weeks. This is comparable with pCR rates of $4.4 \%-$ $25 \%$ in literature, with intervals to surgery varying from 4 to 19 weeks [9,32-40]. However, there were some differences in tumor characteristics between the groups in our dataset. Furthermore, we cannot relate these outcomes to local recurrence rates or survival. Three-year OS of $73-78 \%$ vs. $65-82.4 \%$ and DFS of $53-59 \%$ vs. $52-75.1 \%$ have been previously described for SCRT-delay and CRT, respectively [9,32]. However, these studies included younger, WHO 0-1 patients. Differences in survival are partly determined by differences in patient selection for surgical treatment and choices in management of older patients with colorectal cancer might greatly affect population-based survival [41]. Also, the majority of these patients received adjuvant chemotherapy. This is not a part of routine care in the Netherlands.

The post-matching cohort represents a comparison of SCRT-delay and CRT in two groups with equivalent age and comorbidities. Here we did not find a difference in postoperative complications nor in pathological outcomes between SCRT-delay and CRT. These results are in line with 2 randomized trials comparing SCRTdelay \pm chemotherapy and CRT [9,32]. Also, in the Stockholm III trial the frequency of postoperative complications decreased by delaying surgery with $4-8$ weeks after SCRT $[8,12]$. This indicates that, considering surgery-related complications and pathological outcomes, SCRT-delay could be a good alternative neoadjuvant treatment option for LARC patients who are unable to undergo CRT. However, information on treatment compliance is lacking from this study. In the Stockholm III trial, 7\% of patients were hospitalized for radiation 
Table 3

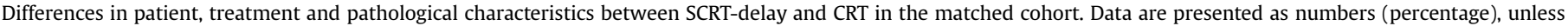
stated otherwise.

$\begin{array}{lll}\text { SCRT-delay } & \text { CRT } & \text { p-value } \\ n=246 & n=246 & \end{array}$

\section{Patient characteristics}

Gender

Male

$142(57.7)$

104 (42.3)

Age (mean years (sd))

BMI (mean $\left.\mathrm{kg} / \mathrm{m}^{2}(\mathrm{sd})\right)$

Comorbidities

ASA score

1

2

3

4

Tumor characteristics

$162(65.9)$

$84(34.1)$

0.078

Distance from anus at colonoscopy (mean $\mathrm{cm}(\mathrm{sd})$ )

cT
2

3

Distance to MRF $>1 \mathrm{~mm}$

cN

1

Diverting colostomy

Permanent colostomy

\section{Pathological characteristics}

pT

1

2

4

0
1
2

$x$
$76.72(8.86)$

25.51 (3.96)

$208(84.6)$

$20(8.1)$
$132(53.7)$
$87(35.4)$
$7(2.8)$

75.90 (8.39)

$26.30(3.91)$

223 (90.7)

5.91 (4.17)

7 (2.8)

$190(77.2)$

49 (19.9)

52 (21.1)

61 (24.8)

68 (27.6)

115 (46.7)

$2(0.8)$

$11.00[9.00,15.00]$

48 (19.5)

$179(72.8)$

17 (6.9)

$2(0.8)$

$2(0.8)$

$133(54.1)$

$110(44.7)$

$1(0.4)$

33 (13.4)

5 (2.0)

$24(9.8)$

4 (1.6)

$1(0.4)$

0

1 (0.4)

$3(1.2)$

$1(0.4)$

$1(0.4)$

$2(0.8)$

57 (23.2)

50 (20.3)

$25(10.2)$

$1(0.4)$

7 (2.8)

163 (66.3)

$23(9.3)$
$12(4.9)$
$64(26)$
$131(53.3)$
$16(6.5)$

$154(62.6)$
$59(24)$
$32(13)$
0

23 (9.3)

$4(26)$

$131(53.3)$

6 (6.5)

59 (24)

0

$18(7.3)$

152 (61.8)

74 (30.1)

2 (0.8)

$6.62(4.54)$

0.071

9 (3.7)

182 (74)

55 (22.4)

61 (24.8)

0.123

37 (15)

69 (28)

138 (56.1)

$2(0.8)$

0.294

0.027 *

0.091

0.148

0.681

0.047 *

$11.00[10.00,13.00]$

0.361

0.660

54 (22)

$175(71.1)$

13 (5.3)

4 (1.6)

4 (1.6)

128 (52)

$113(45.9)$

$1(0.4)$

33 (13.4)

$10(4.1)$

$21(8.5)$

$2(0.8)$

$1(0.4)$

$1(0.4)$

$2(0.8)$

$2(0.8)$

$1(0.4)$

$1(0.4)$

$1(0.4)$

73 (29.7)

0.125

34 (13.8)

$42(17.1)$

4 (1.6)

8 (3.3)

158 (64.2)

0.849

1.000

0.469

0.967

0.054

38 (15.4)

14 (5.7)

62 (25.2)

115 (46.7)

17 (6.9)

168 (68.3)

48 (19.5)

27 (11)

1 (0.4)

Please cite this article as: Hoendervangers $S$ et al., The effect of neoadjuvant short-course radiotherapy and delayed surgery versus chemoradiation on postoperative outcomes in locally advanced rectal cancer patients - A propensity score matched nationwide audit-based study, European Journal of Surgical Oncology, https://doi.org/10.1016/j.ejso.2020.03.002 


\begin{tabular}{|c|c|c|c|}
\hline & SCRT-delay & CRT & p-value \\
\hline & $\mathrm{n}=246$ & $\mathrm{n}=246$ & \\
\hline Pathological complete response, ypTONO & $19(7.7)$ & $31(12.6)$ & 0.101 \\
\hline Radicality (RO resection) & $226(91.9)$ & $219(89)$ & 0.357 \\
\hline
\end{tabular}

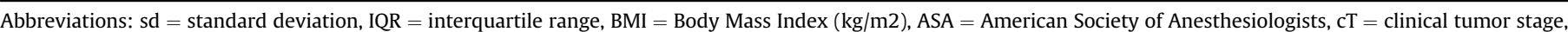

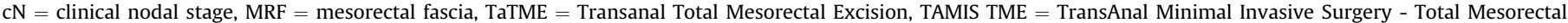
Excision, $\mathrm{pT}=$ pathological tumor stage, $\mathrm{pN}=$ pathological nodal stage .

Table 4

Differences in postoperative outcomes between SCRT-delay and CRT in the matched cohort. Data are presented as numbers (percentage), unless stated otherwise.

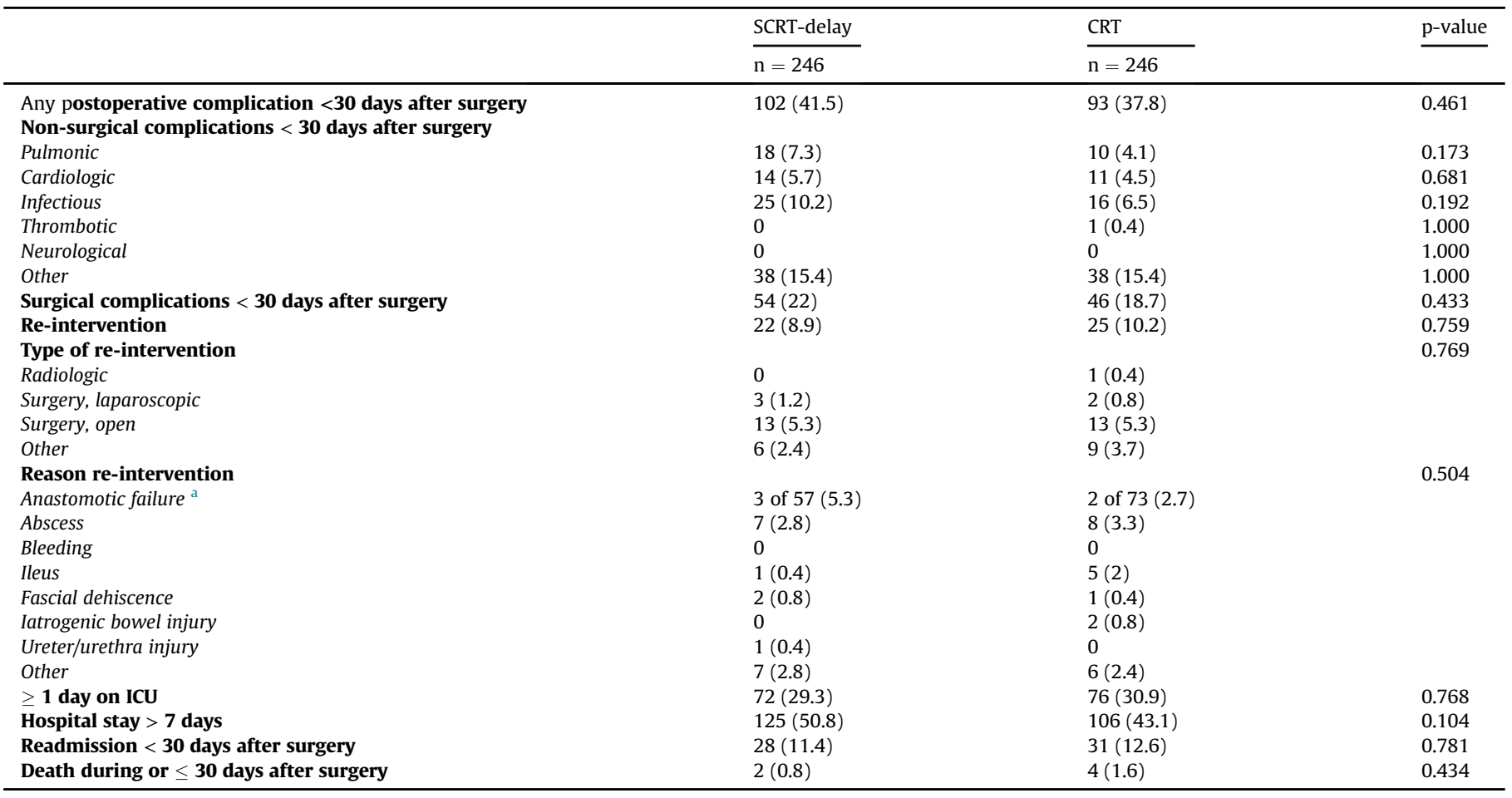

Abbreviations: ICU = intensive care unit.

a Data shown for patients that received a primary anastomosis.

toxicity. Previous studies suggest that compliance and immediate toxicity are in favor of SCRT (compared to CRT) [1], but more data is needed. Furthermore, long-term outcomes on local recurrence and survival is needed.

This is the first observational study that compares complications after SCRT-delay and CRT in a large population. Since observational studies cannot determine treatment effects as accurately as randomized trials [22], this propensity score matched study may provide a useful estimation of the differences between SCRT-delay and CRT. Nonetheless, the results of this study should be interpreted carefully. Confounding bias is frequently seen in observational studies $[42,43]$. Patient and disease characteristics may have influenced the selection of patients for neoadjuvant and surgical treatment. Most likely, only well-conditioned patients are included in this database. The biggest pitfall of this study, however, is confounding by indication, since the selection of neoadjuvant treatment is confounded by patient factors, which are also related to the outcome [44,45]. Adjusting for confounding by indication using propensity score analysis is reliable when data on all factors associated with the intervention and the outcome is precise and can be accounted for $[23,44]$. However, unadjusted confounding may still exist if unmeasured factors influenced treatment selection. This may lead to biased results $[22,23,46]$.
The aging population, the rising incidence and the improved prognosis of rectal cancer will increase the need for surgery in the elderly population in the future $[4,26]$. Successful treatment of elderly patients depends on whether it is done safely, allowing them to preserve good quality of life, and a life-expectancy that is not reduced by the treatment [2]. Regarding surgery-related complications, SCRT-delay is a good alternative neoadjuvant treatment option for frail LARC patients. However, information on treatment compliance and quality of life is needed. Secondly, before the indication for SCRT-delay can be expanded to intermediate risk rectal cancer or high risk rectal cancer in the general population, more data on long-term outcomes, such as local recurrence and survival, is needed.

\section{Disclosures and/or funding}

None.

This paper is not based on a previous communication to a society or meeting.

\section{CRediT authorship contribution statement}

S. Hoendervangers: Conceptualization, Methodology, Formal 
analysis, Writing - original draft, Writing - review \& editing. C.L. Sparreboom: Conceptualization, Methodology, Writing - original draft, Writing - review \& editing. M.P.W. Intven: Writing - review \& editing, Supervision. J.F. Lange: Supervision, Writing - review \& editing. H.M. Verkooijen: Conceptualization, Methodology, Writing - review \& editing, Supervision. P.G. Doornebosch: Conceptualization, Writing - review \& editing, Supervision. W.M.U. van Grevenstein: Conceptualization, Writing - review \& editing, Supervision.

\section{Acknowledgements}

The authors would like to thank all surgeons and other health care providers who are involved in registering patients in the Dutch ColoRectal Audit (DCRA).

\section{Appendix A. Supplementary data}

Supplementary data to this article can be found online at https://doi.org/10.1016/j.ejso.2020.03.002.

\section{References}

[1] Lutz MP, et al. Second st. Gallen European organisation for research and treatment of cancer gastrointestinal cancer conference: consensus recommendations on controversial issues in the primary treatment of rectal cancer. Eur J Canc 2016;63:11-24.

[2] Surgery for colorectal cancer in elderly patients: a systematic review. Colorectal Cancer Collaborative Group. Lancet 2000;356(9234):968-74.

[3] Manceau G, et al. Comparative outcomes of rectal cancer surgery between elderly and non-elderly patients: a systematic review. Lancet Oncol 2012;13(12):e525-36.

[4] Hathout L, et al. Management of locally advanced rectal cancer in the elderly: a critical review and algorithm. J Gastrointest Oncol 2018;9(2):363-76.

[5] Verweij NM, et al. Colorectal cancer resections in the oldest old between 2011 and 2012 in The Netherlands. Ann Surg Oncol 2016;23(6):1875-82.

[6] Montroni I, et al. Personalized management of elderly patients with recta cancer: expert recommendations of the European society of surgical oncology, European society of coloproctology, international society of geriatric oncology, and American college of surgeons commission on cancer. Eur J Surg Oncol 2018:44(11):1685-702.

[7] Bujko K, et al. Long-term results of a randomized trial comparing preoperative short-course radiotherapy with preoperative conventionally fractionated chemoradiation for rectal cancer. Br J Surg 2006;93(10):1215-23.

[8] Erlandsson J, et al. Optimal fractionation of preoperative radiotherapy and timing to surgery for rectal cancer (Stockholm III): a multicentre, randomised, non-blinded, phase 3, non-inferiority trial. Lancet Oncol 2017;18(3):336-46.

[9] Latkauskas $T$, et al. Preoperative conventional chemoradiotherapy versus short-course radiotherapy with delayed surgery for rectal cancer: results of a randomized controlled trial. BMC Canc 2016;16(1):927.

[10] Fokstuen T, Holm T, Glimelius B. Postoperative morbidity and mortality in relation to leukocyte counts and time to surgery after short-course preoperative radiotherapy for rectal cancer. Radiother Oncol 2009;93(2):293-7.

[11] Sparreboom CL, et al. Anastomotic leakage and interval between preoperative short-course radiotherapy and operation for rectal cancer. J Am Coll Surg 2018;227(2):223-31.

[12] Erlandsson J, et al. Postoperative complications in relation to overall treatment time in patients with rectal cancer receiving neoadjuvant radiotherapy. Br J Surg 2019 Aug;106(9):1248-56.

[13] Van Leersum NJ, et al. The Dutch surgical colorectal audit. Eur J Surg Oncol 2013;39(10):1063-70.

[14] Lefevre $\mathrm{JH}$, et al. Effect of interval ( 7 or 11 weeks) between neoadjuvant radiochemotherapy and surgery on complete pathologic response in rectal cancer: a multicenter, randomized, controlled trial (GRECCAR-6). J Clin Oncol 2016;34(31):3773-80.

[15] Charlson ME, et al. A new method of classifying prognostic comorbidity in longitudinal studies: development and validation. J Chron Dis 1987;40(5): $373-83$.

[16] Moons KG, et al. Using the outcome for imputation of missing predictor values was preferred. J Clin Epidemiol 2006;59(10):1092-101.

[17] van Buuren S. Mice: multivariate imputation by chained equations in R. J Stat Software 2011:45(3)

[18] Bodner TE. What improves with increased missing data imputations? Struct
Equ Model Multidiscipl J 2008;15(4):651-75.

[19] White IR, Royston P. Wood AM. Multiple imputation using chained equations: issues and guidance for practice. Stat Med 2011;30(4):377-99.

[20] Rosenbaum PR, Rubin DB. Reducing bias in observational studies using subclassification on the propensity score. J Am Stat Assoc 1984;79(387):516-24.

[21] Austin PC. Optimal caliper widths for propensity-score matching when estimating differences in means and differences in proportions in observational studies. Pharmaceut Stat 2011;10(2):150-61.

[22] Haukoos JS, Lewis RJ. The propensity score. J Am Med Assoc 2015;314(15): 1637-8.

[23] Lonjon G, et al. Potential pitfalls of reporting and bias in observational studies with propensity score analysis assessing a surgical procedure: a methodological systematic review. Ann Surg 2017;265(5):901-9.

[24] Austin PC. Using the standardized difference to compare the prevalence of a binary variable between two groups in observational research. Commun Stat Simulat Comput 2009;38(6):1228-34.

[25] Gietelink L, et al. Changes in nationwide use of preoperative radiotherapy for rectal cancer after revision of the national colorectal cancer guideline. Eur Surg Oncol 2017;43(7):1297-303.

[26] Rutten $\mathrm{HJ}$, et al. Controversies of total mesorectal excision for rectal cancer in elderly patients. Lancet Oncol 2008;9(5):494-501.

[27] Schiphorst AH, et al. Age-related guideline adherence and outcome in low rectal cancer. Dis Colon Rectum 2014:57(8):967-75.

[28] Shahir MA, et al. Elderly patients with rectal cancer have a higher risk of treatment-related complications and a poorer prognosis than younger patients: a population-based study. Eur J Canc 2006;42(17):3015-21.

[29] Hain E, et al. Bowel dysfunction after anastomotic leakage in laparoscopic sphincter-saving operative intervention for rectal cancer: a case-matched study in 46 patients using the Low Anterior Resection Score. Surgery 2017:161(4):1028-39.

[30] Mongin C, et al. Does anastomotic leakage impair functional results and quality of life after laparoscopic sphincter-saving total mesorectal excision for rectal cancer? A case-matched study. Int J Colorectal Dis 2014;29(4):459-67.

[31] Govaert JA, et al. Nationwide outcomes measurement in colorectal cance surgery: improving quality and reducing costs. J Am Coll Surg 2016;222(1): $19-29$ e2.

[32] Bujko $\mathrm{K}$, et al. Long-course oxaliplatin-based preoperative chemoradiation versus 5 x 5 Gy and consolidation chemotherapy for cT4 or fixed cT3 recta cancer: results of a randomized phase III study. Ann Oncol 2016;27(5): $834-42$.

[33] Chung MJ, et al. Preoperative short- vs. long-course chemoradiotherapy with delayed surgery for locally advanced rectal cancer. Oncotarget 2016 Jun 24;8(36):60479-86.

[34] Faria S, et al. Phase II trial of short-course radiotherapy followed by delayed surgery for locoregionally advanced rectal cancer. Colorectal Dis 2014;16(2): 066-70.

[35] Hatfield P, et al. Short-course radiotherapy, with elective delay prior to surgery, in patients with unresectable rectal cancer who have poor performance status or significant co-morbidity. Radiother Oncol 2009;92(2):210-4.

[36] Hoendervangers $S$, et al. Comparison of pathological complete response rates after neoadjuvant short-course radiotherapy or chemoradiation followed by delayed surgery in locally advanced rectal cancer. Eur J Surg Oncol 2018;44(7):1013-7.

[37] Myerson RJ, et al. Five fractions of radiation therapy followed by 4 cycles of FOLFOX chemotherapy as preoperative treatment for rectal cancer. Int I Radiat Oncol Biol Phys 2014;88(4):829-36.

[38] Pach R, et al. Randomized clinical trial on preoperative radiotherapy 25 Gy in rectal cancer-treatment results at 5-year follow-up. Langenbeck's Arch Surg 2012;397(5):801-7.

[39] Pettersson D, et al. Tumour regression in the randomized Stockholm III Trial of radiotherapy regimens for rectal cancer. Br J Surg 2015;102(8):972-8. discussion 978.

[40] Radu C, et al. Short-course preoperative radiotherapy with delayed surgery in rectal cancer - a retrospective study. Radiother Oncol 2008;87(3):343-9.

[41] Benitez Majano S, et al. Surgical treatment and survival from colorectal cancer in Denmark, England, Norway, and Sweden: a population-based study. Lancet Oncol 2019;20(1):74-87.

[42] Groenwold RH, Hoes AW, Hak E. Confounding in publications of observational intervention studies. Eur J Epidemiol 2007:22(7):413-5.

[43] Groenwold RHH, et al. Selection of confounding variables should not be based on observed associations with exposure. Eur J Epidemiol 2011;26(8):589-93.

[44] Grobbee DE, Hoes AW. Confounding and indication for treatment in evaluation of drug treatment for hypertension. Br Med J 1997;315(7116):1151-4.

[45] Hak E, et al. Confounding by indication in non-experimental evaluation of vaccine effectiveness: the example of prevention of influenza complications J Epidemiol Community Health 2002;56(12):951-5.

[46] Brookhart MA, et al. Variable selection for propensity score models. Am J Epidemiol 2006;163(12):1149-56. 